\title{
Optical strain sensor based on FPI micro-cavities produced by the fiber fuse effect
}

\author{
M. Fátima Domingues ${ }^{\mathrm{a}, *}$, Paulo Antunes ${ }^{\mathrm{a}}$, Nélia Alberto $^{\mathrm{b}}$, Rita Frias ${ }^{\mathrm{a}}$, Rute A. S. Ferreira ${ }^{\mathrm{c}}$, \\ Paulo André ${ }^{\mathrm{d}}$ \\ ${ }^{a}$ Instituto de Telecomunicações and Departamento de Física da Universidade de Aveiro, 3810-193 \\ Aveiro, Portugal \\ ${ }^{b}$ Instituto de Telecomunicações and TEMA - NRD, Mechanical Engineering Department and Aveiro \\ Institute of Nanotechnology, University of Aveiro, 3810-193 Aveiro, Portugal \\ 'Department of Physics and CICECO Universidade de Aveiro, 3810-193 Aveiro, Portugal \\ ${ }^{\mathrm{d}}$ Instituto de Telecomunicações and Department of Electronic and Computer Engineering, Instituto \\ Superior Técnico, University of Lisbon, 1049-001 Lisboa, Portugal
}

\begin{abstract}
In this work we present a cost effective strain sensor based on micro-cavities produced through the re-use of optical fibers destroyed by the catastrophic fuse effect. The strain sensor estimated sensitivity is $2.22 \pm 0.08 \mathrm{pm} / \mu \varepsilon$. After the fuse effect, the damaged fiber becomes useless and, consequently, it is an economical solution for sensing proposes, when compared with the cavities produced using other complex methods. Also, the low thermal sensitivity is of great interest in several practical applications, allowing eluding cross-sensitivity with less instrumentation, and consequently less cost.
\end{abstract}

Keywords: Fiber optics, Fiber fuse effect, Optical sensors, Fabry-Perot Interferometer, Strain.

\section{INTRODUCTION}

Strain sensors are widely used for a large variety of applications such as aerospace, automotive and motor sports, dental and medical sensors, biometrics, structural health monitoring of engineering structures, among many others [1-3].

On the last decades, due to considerable research investments worldwide, optical fiber sensors appeared as one of the most promising sensing technologies due to its advantages over traditional electronic sensing. Namely, its reduced weight and volume, immunity to electromagnetic interference, electrical isolation (don't make use of any electrical signals) makes them explosion safe with no interference from electromagnetic radiation.

The need for sensors to ensure greater reliability and security in high risk locations, such as oil rigs and nuclear plants, has significantly motivated the research on this kind of sensors. Among those, the Fiber Bragg gratings (FBG) or FabryPerot Interferometer (FPI) micro-cavities based sensors have become the most attractive due to the miniature size, linear response and higher sensitivity $[1,4-6]$. Nevertheless, its production requires a high economical investment and complex assembling techniques.

In this work we present a novel and cost effective technique to develop optical fiber FPI strain sensors, based on the recycling of optical fiber destroyed by the catastrophic fuse effect. This phenomenon, first observed in 1987 [7], is characterized by the continuous self-destruction of the optical fiber, which is induced by a high intensity optical signal. This singularity can be initiated by a local heating point, frequently a damaged or unclean connector, or in a fiber tight bend [8].

*fdomingues@av.it.pt; phone +351234 377 900, Fax: +351 234377901.

23rd International Conference on Optical F bre Sensors, edited by José Miguel López-Higuera,

Julian Jones, Manuel López-Amo, José Luis Santos, Proc. of SPIE Vol. 9157, 91571Q

(C) 2014 SPIE - CCC code: 0277-786X/14/\$18 - doi: 10.1117/12.2059645 


\section{SENSOR PRODUCTION AND EXPERIMENTAL CHARACTERIZATION}

After the catastrophic fuse effect propagation, the damaged optical fibers shown periodic voids in the core region, whose dimensions and spatial period are in the order of a few micrometers [9], as illustrated in Figure 1.

In this way, the damaged fiber becomes useless and, consequently, using it for sensing proposes and taking advantage of the micro-structures created naturally, is an economical solution when compared with other techniques. The sensing micro-cavity is obtained by splicing a damaged fiber, with a standard optical fiber.

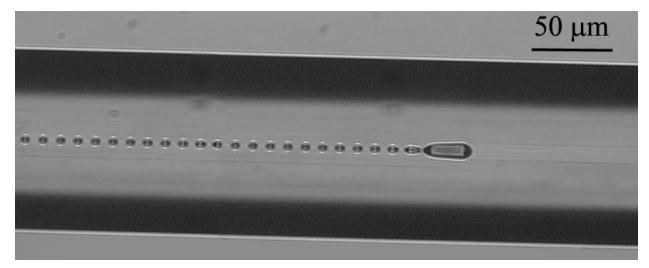

Figure 1. Fiber fuse effect voids and end point.

Once the fuse effect damaged optical fiber is cleaved on the end void, the fiber is placed on a splicing machine from Fujikura, model FSM-040S, allowing the alignment and the splice of both fibers with an electrical discharge, as demonstrated in Figure 2 sequence. The fibers alignment is made through the cladding and the discharge time is $2000 \mathrm{~ms}$.
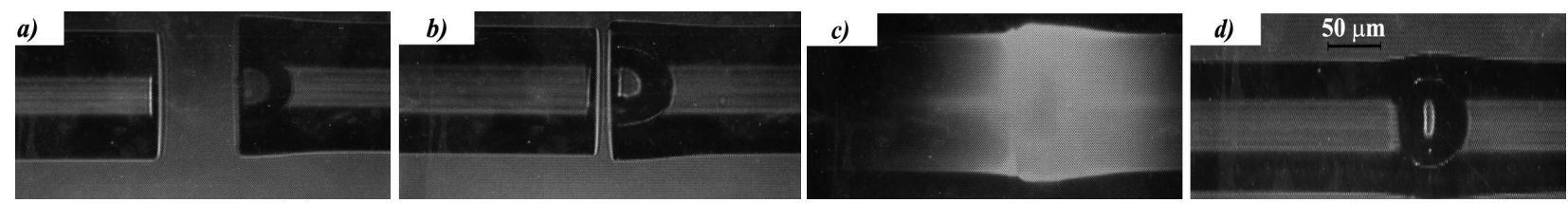

Figure 2. Splicing process images from the splicing machine: a) positioning, b) alignment, c) electrical discharge and d) final result.

According to the method presented, an optical FPI micro-cavity sensor was manufactured, as shown in Figure 3, left.

In order to characterize the sensor to mechanical perturbations, a linear translation stage with a micrometric screw, for control, was used, Figure 3, right. The fiber, containing the micro-cavities was fixed with epoxy to a rigid support and the linear translation stage, and a 24 hours' time interval was given for curing. The distance between fixation points was $12.84 \mathrm{~mm}$. After curing, the test was performed, applying an increasing longitudinal strain to the fibers. The optical spectrum was obtained for each sensor, on an imposed elongation interval ranging from $0 \mu \mathrm{m}$ to $140 \mu \mathrm{m}$, in $10 \mu \mathrm{m}$ steps. The monitoring data was acquired with an interrogation unit from Micron Optics, model sm125, with a resolution of $1.0 \mathrm{pm}$.
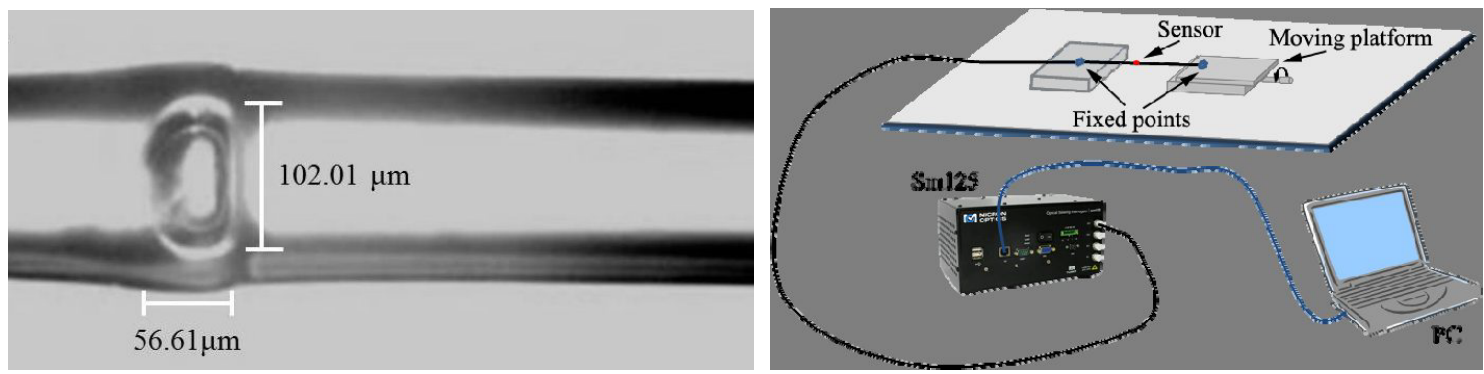

Figure 3. Left: Manufactured optical FPI micro-cavity sensor, with its dimensions; Right: experimental setup.

The obtained optical power spectra, for the FPI sensor are presented in Figure 4 on the left, along with the strain characterization. From the data displayed it's possible to estimate the strain sensitivity of the proposed sensor. Its 
sensitivity is obtained by a linear fit to the wavelength shift as a function of the applied strain, which is represented in Figure 4 , on the right. A strain sensitivity of $2.22 \pm 0.08 \mathrm{pm} / \mu \varepsilon$ was achieved.
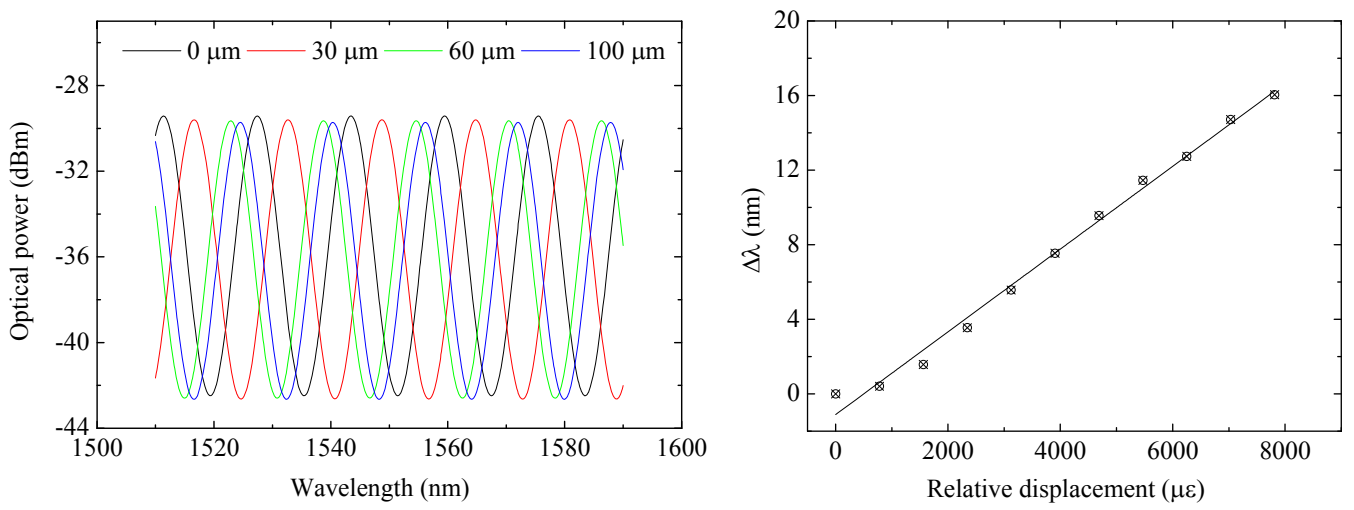

Figure 4. Left: Measured spectra for different longitudinal strain levels; Right: measured wavelength shift with the applied strain. The dots represent the experimental data and the line the linear fit.

Regarding the thermal characterization, it was performed placing the sensors inside a climatic chamber, Model 340 from the Challenge Angelantoni Industrie, and varying the temperature in an interval ranging from $0{ }^{\circ} \mathrm{C}$ to $100{ }^{\circ} \mathrm{C}$ in $20^{\circ} \mathrm{C}$ steps. The measured spectra for different temperatures are presented on Figure 5.

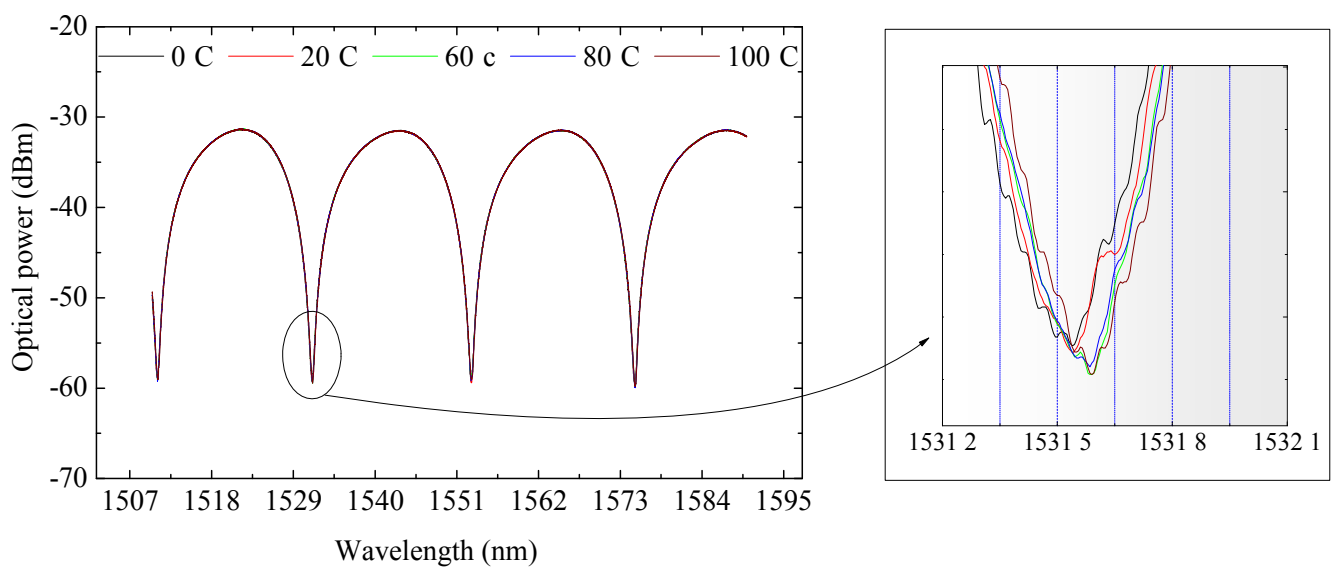

Figure 5. Measured spectra for different temperatures.

Figure 5 shows a typical interference fringe with a temperature red shift observed for the entire optical spectrum. The measured wavelength shift for different temperatures leads to a thermal sensitivity of $3.7 \pm 2.1 \mathrm{pm} /{ }^{\circ} \mathrm{C}$.

A FPI is in general composed by two parallel reflecting surfaces separated by a certain distance. The interference occurs due to the multiple super-positions of the reflected and transmitted radiation at the surfaces. The optical phase difference between the reflected or transmitted light beams causes a wavelength intensity modulation on the reflection spectrum of an optical FPI cavity. The phase difference of the optical FPI $\left(\phi_{F P I}\right)$ can be expressed as [4]:

$$
\phi_{F P I}=\frac{2 \pi}{\lambda} n 2 L
$$

Where $\lambda$ is the wavelength of the incident light, $n$ is the refractive index of cavity material and $L$ is the physical length of the cavity.

If a fiber with a cavity is subjected to a physical perturbation, the phase difference will change with the variation of the optical path length difference and the wavelength shift on the FPI cavity can be related with the longitudinal relative 
elongation, $\varepsilon_{z}$, according with the following expression:

$$
\frac{d \lambda_{m}}{d S}=\frac{4}{(2 m+1)} L \varepsilon_{\mathrm{z}}
$$

where $\lambda_{m}$ is the wavelength of the $m^{\text {th }}$ order interference mode, $m$ an integer and $L$ the cavity length. The micro-cavity radial dimension is greater than the optical fiber core diameter, allowing a large number of transversal optical modes to be transmitted. Additionally, due to the comparable diameter of the fiber with the cavity width, a longitudinal axial strain also imposes an alteration (decrease) of the cavity width. The micro-cavity unveils an ellipsoid contour, thus, it is expected that the interference condition and the strain sensitivity exhibit dependence with the cavity volume. This fact is often ignored by other authors that apply the traditional FPI model to micro-cavities [5]. Even models developed to spheroidal Fabry-Perot micro-cavities fail to describe, accurately, the strain sensitivity dependence with the cavity length [10]. Based on the above and considering the cavity parameters measured and a radial symmetry, for the strain value of $2.2 \pm 0.8 \mathrm{pm} . \mu \varepsilon^{-1}$ a cavity volume of $1.388 \times 10^{-6} \mu \mathrm{m}^{3}$ was obtained.

\section{CONCLUSION}

Within this work, we demonstrate a lower cost manufacturing process for FPI micro-cavity sensor, achieving strain and thermal sensitivities similar to previously reported solutions with much higher manufacturing costs and complexity. The sensors manufacturing process allows re-using the otherwise useless standard optical fiber, damaged by the fiber fuse effect. This technique considerably reduces the manufacturing costs and the complexity of the process, when compared to other techniques.

\section{ACKNOWLEDGMENTS}

Fátima Domingues, Nélia Alberto and Paulo Antunes acknowledge Fundação para a Ciência e Tecnologia (FCT) for the Ph.D. fellowship SFRH/BD/69097/2010, and the Postdoctoral fellowships SFRH/BPD/78141/2011, and SFRH/BPD/76735/2011, respectively.

\section{REFERENCES}

[1] Antunes, P., Lima, H., Alberto, N., Pinto, P., Costa, A. Rodrigues, Pinto, J., Nogueira, R., Varum. H. and André, P., [New Developments in Sensing Technology for Structural Health Monitoring], Springer, New York, 253-295 (2011).

[2] Lee, B., "Review of the present status of optical fiber sensors," Opt. Fiber Technol. 9, 57-79 (2003).

[3] Leitão, C., Bilro, L., Alberto, N., Antunes, P., Lima, H., André, P. S., Nogueira, R., and Pinto, J. L., "Feasibility studies of Bragg probe for noninvasive carotid pulse waveform assessment," J. Biomed. Opt. 18, 017006-017006 (2013).

[4] Lee, B. H., Kim, Y. H., Park, K. S., Eom, J. B., Kim, M. J., Rho, B. S., and Choi, H. Y., "Interferometric fiber optic sensors," Sensors 12, 2467-2486 (2012).

[5] Liao, C. R., Hu, T. Y., and Wang, D. N., "Optical fiber Fabry-Perot interferometer cavity fabricated by femtosecond laser micromachining and fusion splicing for refractive index sensing," Opt. Express 20, 22813-22818 (2012).

[6] Antunes, P.F.C.; Domingues, M.F.F.; Alberto, N.J.; Andre, P.S., "Optical fiber microcavity strain sensors produced by the catastrophic fuse effect," Photon. Technol. Lett. 26 (1), 78-81 (2014).

[7] Kashyap, R. and Blow, K. J., "Observation of catastrophic self-propelled self-focusing in optical fibers," Electron. Lett. 24, 47-49 (1988).

[8] Domingues, F., Rocha, A. M., and Andre, P. S., "High-power effects in damaged and contaminated optical fiber connectors," Microw. Opt. Technol. Lett. 53, 2485-2488 (2011).

[9] Domingues, F., Frias, A.R., Antunes, P., Sousa, A.O.P., Ferreira, R.A.S. and Andre, P. S., "Observation of fuse effect discharge zone nonlinear velocity regime in erbium-doped fibres," Electron. Lett. 48, 1295-1296 (2012).

[10] Favero, F. C., Araujo, L., Bouwmans, G., Finazzi, V., Villatoro, J., and Pruneri,V., "Spheroidal Fabry-Perot microcavities in optical fibers for high-sensitivity sensing," Opt. Express 20, 7112-7118 (2012). 